\title{
Unknown primary tumors: is there a future therapeutic role for immune checkpoint inhibitors?
}
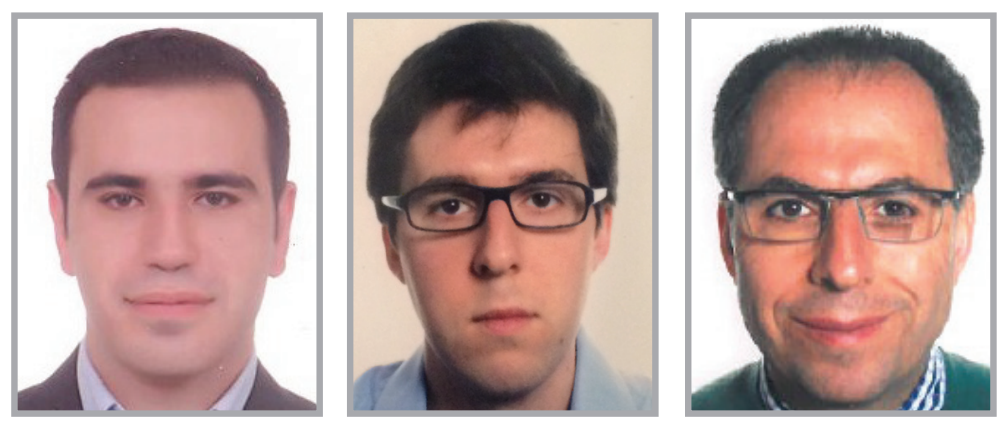

Hampig Raphael Kourie* ${ }^{*, 1}$, Gil Awada² \& Ahmad Hussein Awada ${ }^{1}$

First draft submitted: 17 November 2015; Accepted for publication: 24 November 2015; Published online: 18 January 2016

Cancers of unknown primary (CUP) or occult primary tumors are defined as histologically proven metastatic malignant tumors, whose primary site cannot be identified during pretreatment evaluation [1]. Different hypotheses can explain the phenomenon of not finding the primary cancer: small and slowly growing primary, disappearance of the primary because of an immunologic response or less probably a removed primary during surgery for another condition [2].

Determining the accurate treatment for CUP is actually based on the histologic subtype of the tumor, the localization of the metastasis, the sex and the age of the patient [3]. After negative initial evaluation and complete work-up including blood tests, imaging (including PET/computed tomography) and endoscopic exams, the pathologic diagnosis on biopsies can rule out first lymphoma and other malignancies, melanoma, thyroid carcinoma, sarcoma, germ cell tumors and nonmalignant diagnosis. Epithelial not site-specific malignancies can be classified into four histologic subtypes : adenocarcinoma, squamous, neuroendocrine and not otherwise specified [4]. Of note, tumor gene profiling did not emerge as a tool to precise the type of primary tumor. For example, the treatment of adenocarcinoma of unknown primary is first determined according to the localization of the tumor, then to the sex and the age. Thus, an axillary adenocarcinoma in women is usually treated as a breast cancer, while an adenocarcinoma of the mediastinum is treated as poor-risk germ cell tumor in patients aged less than 40 years and as non-small-cell lung cancer (NSCLC) in those aged more than 50 years.

Different chemotherapeutical regimens are indicated in the treatment of tumors of unknown primary [5]. These regimens are chosen to have the same potential of efficacy in the different possibilities of the eventual primary.

In the mid-1990s, two immunological modulating agents, IL-2 and IFN, were

\section{KEYWORDS}

- cancer • checkpoint inhibitors

- clinical trial • diagnosis • future

- immunotherapies

- ipilimumab • nivolumab

- pembrolizumab • unknown primary tumor

“Different hypotheses can explain the phenomenon of not finding the primary cancer...” 
“...it will be rational to launch a prospective study first, to evaluate the potential effect of checkpoint inhibitors in carcinoma of unknown primary." approved in the treatment of melanoma and renal cell carcinoma; these drugs presented a low response rate with major side effects. In the new era of immunotherapies, checkpoint inhibitors are becoming the 'magic drugs' with remarkable response rates, acceptable side effects and acting on different cancer types. The most studied, evaluated and consequently approved checkpoint inhibitors are anti-CTLA4 and anti-PD-1 and its ligand (anti-PD-L1) agents. Checkpoint inhibitors act by blocking inhibitory receptors of immune system elements (CTLA4, PD-1 and its ligand PD-L1), leading to the activation of tumorspecific $T$ cells with effector function against tumor cells [6].

Ipilimumab (anti-CTLA4) was first approved by the US FDA in 2011 as first-line treatment for unresectable or metastatic melanoma. Anti-PD-1 agents were approved in melanomas and squamous NSCLC [7]. Thus, pembrolizumab and nivolumab were approved in 2014 as second-line treatment for unresectable or metastatic melanoma progressing following ipilimumab and, if BRAF V600-mutation positive, a BRAF inhibitor $[8,9]$. Nivolumab was approved for metastatic squamous NSCLC progressing on or after a platinum-based chemotherapy, in April 2015 and this approval was expanded for NSCLC with non-squamous histology [10,11]. Pembrolizumab was also approved in October 2015 for the treatment of patients with metastatic NSCLC whose tumors express PD-L1, with disease progression on or after platinum-containing chemotherapy [12]. For the first time, a combination of checkpoint inhibitors, nivolumab and ipilimumab was approved for the treatment of patients with $B R A F$ V600 wild-type, unresectable or metastatic melanoma [13] and for the first time Ipilimumab was approved in the adjuvant setting in melanomas. A Phase III trial comparing nivolumab to everolimus as second-line treatment in metastatic renal cell carcinoma showed positive results, waiting the official approval of nivolumab by FDA in this indication [14]. Several ongoing trials testing checkpoint inhibitors are being evaluated in head and neck cancers, bladder cancer, lung cancer, breast cancer, ovarian cancer, prostate cancer and hematologic malignancies with early promising results. Anti-PD-1 agents are presenting acceptable response rates (RR) in different malignancies in Phase I and II trial, those RR varies from
$11.5 \%$ in ovarian cancer [15], $20 \%$ in gastric cancer [16], 24\% in urothelial cancer [17], 15\% in small-cell lung cancer [18], 40\% in colorectal cancer with mismatch repair deficiency [19] and $80 \%$ in Merckel cell carcinoma [20].

Since these new agents have a potential efficacy in different cancer types, they can be considered in carcinomas of unknown primary. For example, an old man presenting a pelvic not otherwise determined carcinoma of unknown primary; the most frequent incriminated primaries are bladder, prostate or kidney cancers. In all those mentioned eventualities, checkpoint inhibitors (especially anti-PD-1/PD-L1) showed a potential benefit. Thus, considering these agents in the panel of therapies of pelvic not otherwise determined carcinoma of unknown primary, next to chemotherapeutical regimens, is a rational choice.

Actually, according to the National Comprehensive Cancer Network guidelines of CUP, adenocarcinoma of axillary nodes in woman is treated as a breast cancer and peritoneal nodules and ascites are treated as ovarian carcinoma. In these cases, checkpoint inhibitors can be considered as second- or third-line treatment, if the first hypothesis seemed not to be plausible, since these agents showed a promising results in triplenegative breast cancer and ovarian carcinoma. In pulmonary nodules of unknown primary, referring patients to clinical trials is the standard of care. Clinical trials evaluating checkpoint inhibitors can be considered in this particular case.

In the emerging era of modern immunotherapies and the important number of settings where these drugs are studied and potentially active, it will be rational to launch a prospective study first, to evaluate the potential effect of checkpoint inhibitors in carcinoma of unknown primary. It will be of interest to design a multicentric randomized trial comparing the efficacy of one of these new immunotherapies (in particular, anit-PD-1/PD-L1) to the traditional chemotherapeutical regimen of physician's choice in the treatment of this subgroup of CUP in the early lines of metastatic disease. Meanwhile, the adoption of this new concept in the treatment of uncontrolled carcinoma of unknown primary can be considered an investigational approach option, waiting the implementation of this therapeutic modality into clinical practice after positive results from prospective clinical trials. 
Financial \& competing interests disclosure

The authors have no relevant affiliations or financial involvement with any organization or entity with a financial interest in or financial conflict with the subject matter or materials discussed in the manuscript. This includes employment, consultancies, honoraria, stock ownership or options, expert testimony, grants or patents received or pending, or royalties.

No writing assistance was utilized in the production of this manuscript.

\section{References}

1 Greco FA, Hainsworth JD. Tumors of unknown origin. CA Cancer J. Clin. 2, 96-115 (1992).

2 Stella GM, Senetta R, Cassenti A et al. Cancers of unknown primary origin: current perspectives and future therapeutic strategies. J. Transl. Med. 10, 12 (2012).

3 NCI website, carcinoma of unknown primary. www.cancer.gov/types

4 Kim KW, Krajewski KM, Jagannathan JP et al. Cancer of unknown primary sites: what radiologists need to know and what oncologists want to know. Am. J. Roentgenol. 3, 484-492 (2013).

5 NCCN guidelines of occult primary. www.nccn.org/professionals

6 Pardoll DM. The blockade of immune checkpoints in cancer immunotherapy. Nat. Rev. Cancer. 12, 252-264 (2012).

7 Hodi FS, O'Day SJ, McDermott DF, Weber RW et al. Improved survival with ipilimumab in patients with metastatic melanoma. N. Engl. J. Med. 363, 711-723 (2010).

8 Hamid O, Robert C, Daud A et al. Safety and tumor responses with lambrolizumab (anti-PD-1) in melanoma. N. Engl. J. Med. 369: 134-144 (2013).
Weber JS, D’Angelo SP, Minor D, Hodi FS et al. Nivolumab versus chemotherapy in patients with advanced melanoma who progressed after anti-CTLA-4 treatment (CheckMate 037): a randomised, controlled, open-label, Phase 3 trial. Lancet Oncol. 16, 375-384 (2015).

10 Rizvi NA, Mazières J, Planchard D et al. Activity and safety of nivolumab, an anti-PD-1 immune checkpoint inhibitor, for patients with advanced, refractory squamous non-small-cell lung cancer (CheckMate 063): a Phase 2, single-arm trial. Lancet Oncol. 16, 257-265 (2015).

11 Borghaei H, Paz-Ares L, Horn Let al. Nivolumab versus docetaxel in advanced nonsquamous non-small-cell lung cancer. N. Engl. J. Med. 17: 1627-39 (2015).

12 Garon EB, Rizvi NA, Hui R et al. Pembrolizumab for the treatment of non-small-cell lung cancer. N. Engl. J. Med. 21, 2018-2028 (2015).

13 Larkin J, Chiarion-Sileni V, Gonzalez R et al. Combined nivolumab and ipilimumab or monotherapy in untreated melanoma. N. Engl. J. Med. 1, 23-34 (2015).

14 Motzer RJ, Escudier B, McDermott DF et al. Nivolumab versus everolimus in advanced renal-cell carcinoma. N. Engl. J. Med. 19, 1803-1813 (2015).
15 Hamanishi J, Mandai M, Ikeda T et al. Safety and antitumor activity of anti-PD-1 antibody, nivolumab, in patients with platinumresistant ovarian cancer. J. Clin. Oncol. 33(34), 4015-4022 (2015).

16 Muro, K, Bang, Y, Shankaran, V, Geva, R et al. A Phase $1 \mathrm{~b}$ study of pembrolizumab (Pembro; MK-3475) in patients (Pts) with advanced gastric cancer. Ann. Oncol. 25(5), 1-41(2014).

17 Plimack ER, Gupta S, Bellmunt J et al. A Phase $1 \mathrm{~b}$ study of pembrolizumab (Pembro; MK-3475) in patients (Pts) with advanced urothelial tract cancer. Ann. Oncol. 25(Suppl. 5), v1-v41 (2014)

18 Scott AJ, Bendell JC, Taylor MH et al. Phase I/II study of nivolumab with or without ipilimumab for treatment of recurrent small cell lung cancer (SCLC): CA209-032. J. Clin. Oncol. 33(Suppl.), Abstract 7503 (2015)

19 Le DT, Uram JN, Wang H et al. PD-1 blockade in tumors with mismatch-repair deficiency. N. Engl. J. Med. 26, 2509-2520 (2015).

20 Nghiem, P, Bhatia, S, Daud, A et al. Activity of PD-1 blockade with pembrolizumab as first systemic therapy in patients with advanced Merkel cell carcinoma. Presented at: The European Cancer Congress (ECC 2015). Vienna, Austria, 25-29 September 2015 (Abstract 22LBA). 